\title{
Civilisations
}

Revue internationale d'anthropologie et de sciences

humaines

60-1|2011

Mobilisations et dynamiques identitaires en Amérique andine

\section{Identités collectives à la frontière}

Héritage et échange, idéologie et innovation

\section{Anne-Laure Amilhat Szary}

\section{(2) OpenEdition}

\section{Journals}

Édition électronique

URL : http://journals.openedition.org/civilisations/2896

DOI : $10.4000 /$ civilisations.2896

ISSN : 2032-0442

Éditeur

Institut de sociologie de l'Université Libre de Bruxelles

Édition imprimée

Date de publication : 31 décembre 2011

Pagination : 81-101

ISBN : 2-87263-035-X

ISSN : 0009-8140

Référence électronique

Anne-Laure Amilhat Szary, « Identités collectives à la frontière », Civilisations [En ligne], 60-1 | 2011, mis en ligne le 13 février 2015, consulté le 30 avril 2019. URL : http://journals.openedition.org/ civilisations/2896 ; DOI : 10.4000/civilisations.2896 


\title{
Identités collectives à la frontière \\ Héritage et échange, idéologie et innovation
}

\author{
Anne-Laure AMILHAT SZARY
}

Résumé : Dans un contexte latino-américain où les populations autochtones ont dî attendre la fin $d u X X^{e}$ siècle pour regagner en visibilité, les formulations contemporaines de l'identité collective sont problématiques. L'article questionne la redécouverte d'un ancrage identitaire longtemps nié ou refoulé, dans des territoires frontaliers où le travail des États a longtemps consisté à renforcer le sentiment national au détriment d'autres territorialités. Il analyse comment les interactions entre les mobilisations socio-politiques et les formulations narratives reconstruisent l'identité collective. Dans le cas étudié, l'alliance " Aymaras sin Fronteras " (Aymaras sans frontières), un processus de territorialisation se produit au nom d'une identité ethnique problématique. Les populations concernées se reconnaissent peu dans une initiative politique tirant parti d'une idéologie des espaces frontaliers ouverts qui ne cö̈ncide qu'en apparence les territoires mobiles traditionnels des Andes centrales.

Mots-clés : montagnes, Andes, Bolivie, Chili, Pérou, ruralité, ethnicité, identité, frontières, transfrontalier, territorialisation, projet de territoire, mobilisation collective.

\begin{abstract}
In a Latin American context where the autochthonous populations have had to wait until the end of the $20^{\text {th }}$ century to regain their visibility, the contemporary formulations of collective identity are problematic. In border spaces where the action of the state consisted for years in reinforcing the national sense of belonging to the detriment of other territorialitites, the paper questions the re-discovery of an identity anchoring which had been for long negated or repressed. It analyses how the interactions between socio-political mobilizations and narrative formulations rebuild collective identity. In the study case, the "Aymaras sin Fronteras " alliance (Aymaras without borders), there is an on-going territorization process in the name of a problematic ethnic identity. The concerned populations do not but feebly recognize themselves in a political initiative benefitting from an ideology of open borderlands that is only apparently built on the traditional Central Andes mobile places.
\end{abstract}

Keywords: mountains, Andes, Bolivia, Chile, Peru, rurality, ethnicity, identity, borders, borderlands, territorialisation, territorial collective mobilisation. 
n accuse traditionnellement les frontières coloniales de s'être imposées à un ordre social préexistant sans le respecter, séparant de façon artificielle des groupes ethniques et culturels établis antérieurement. Mais on oublie généralement de préciser que selon les continents, ce processus politique n'a pas soulevé les mêmes enjeux : l'Amérique latine ne comptait au début XIX ${ }^{\mathrm{e}}$ siècle que 20 millions d'habitants sur 22 millions de $\mathrm{km}^{2}$ (62 millions en 1900) et plus de $85 \%$ des frontières ont été tracées dans des zones très faiblement peuplées (un ou deux hab $/ \mathrm{km}^{2}$ ). Ces chiffres n'impliquent pas que les $15 \%$ restant présentent des situations très conflictuelles. Notre expérience de terrain et d'étude de l'évolution contemporaine des frontières latino-américaines révèle cependant que les solidarités transfrontalières n'y sont pas aussi fortes que l'on pourrait le penser en y appliquant un schéma d'analyse classique. Des liens existent, souvent liés aux savoir-circuler des populations des régions considérées, mais dans la plupart des cas, le nationalisme a pris le pas sur les référents identitaires traditionnels.

Nous proposons d'analyser ici la nature des processus de rapprochement ethnique par delà les frontières. Il s'agit de faire la part entre les héritages et leur reformulation, dans le cadre des mobilisations sociales et politiques récentes. Dans certains cas apparaissent des mouvements politiques qui tirent parti de la situation transfrontalière (laquelle est susceptible de rapporter à ses promoteurs des subsides internationaux pour le développement), au nom d'une identité ethnique dans laquelle les populations concernées se reconnaissent peu. C'est donc au travail sur l'évolution du contenu des discours identitaires en situation frontalière que nous souhaitons consacrer cet article.

\section{La frontière des identités collectives : la question des limites épistémologiques}

Si nous avons choisi de répondre à la question de l'institutionnalisation des mouvements ethniques sur la scène politique et sociale dans les pays andins à partir des espaces frontaliers, c'est qu'ils obligent à une prise en compte de dynamiques multiscalaires, permettant une entrée géographique dans une problématique habituellement traitée par l'analyse anthropologique. Admettre un « concept barthien d'ethnicité » (Morin et Saladin d'Anglure 1995), fondé en grande partie sur la territorialisation du groupe, ne résout pas pour autant la question de la définition des identités collectives, comme le soulignait N. Wachtel à l'heure de la redécouverte des mouvements indigénistes. Dans une note consacrée à cette question (Wachtel 1992), il soulevait la question de l'échelle pertinente de définition du groupe qui devait permettre de parler d'un collectif :

Le problème des identités collectives, dans le monde andin, se pose en termes différents selon qu'on l'aborde à partir du présent ou à partir du XVIe siècle. Lorsque les Espagnols envahirent le Tahuantinsuyu, l'Empire inca des quatre quartiers, ils rencontrèrent plusieurs dizaines de formations sociales de dimensions variables, généralement désignées par le terme de naciones que nous ne savons guère comment traduire: chefferies? groupes ethniques? Mais qu'entendre ici par ethnicité ? Du lac Titicaca au lac Poopo, par exemple, les Lupaqas, les Pacajes, les Carangas, les Soras et les Quillacas formaient des unités socio-politiques distinctes (comportant elles-mêmes diverses subdivisions), mais ils parlaient une même langue, l'aymara, et partageaient, semble-t-il, un univers commun de représentations symboliques. [...] On peut supposer que les lignes de plus grand partage passaient, à l'intérieur de l'ensemble aymara, entre les diverses "nations », et à l'intérieur de l'ensemble uru, entre des sous-unités telles que celles des Iru-Itus, des Ochosu-mas, des Quinaquitaras, etc. Or les lignes de 
partage entre différents groupes sociaux ont une intensité plus ou moins forte et les seuils peuvent se déplacer, à divers niveaux, en fonction de la conjoncture historique : au cours de la période coloniale émergent de nouvelles formations sociales, dont la persistance jusqu'à nos jours ne signifie aucunement qu'elles remontent au XVI' siècle.

Cet avant-propos nous permet de poser le problème en termes géopolitiques. Dans une perspective où l'identité se définit par une somme de composants qui se manifestent à travers les différents moment de l'existence, au cours desquels l'individuel et le collectif interagissent de façon complexe, quelle en est la part territoriale ? Pour les saisir, nous proposons d'analyser les recompositions territoriales, c'est-à-dire un ensemble de processus socio-spatiaux qui conduisent à des réagencements administratifs et politiques, mais également sociaux et identitaires. Le travail sur les espaces de frontière politique entre différents pays apparaît dès lors comme révélateur à la fois d'une évolution des composants de l'identité andine et des conditions de la souveraineté des États. Cela nous permet de travailler l'identité collective à partir de son expression collective. Ce faisant, nous espérons pouvoir ainsi échapper à la mise en garde liminaire de N. Wachtel qui invitait à contextualiser le retour récent de l'ethnicité en Amérique latine :

\begin{abstract}
Il convient, en définitive, de distinguer nettement identité ethnique et identité collective : outre que la première risque d'être arbitrairement décrétée, la seconde ne s'étend pas nécessairement aux dimensions d'un groupe ethnique. Ce qu'on a voulu rappeler ici, c'est l'aspect relativement récent, et étroit, des identités collectives telles qu'on peut les observer aujourd'hui dans les Andes méridionales. Si elles dépassent rarement le cadre des communautés indigènes d'origine coloniale, je ne suggère pas pour autant qu'on puisse attribuer un caractère ethnique à chacune de ces communautés. Pas plus qu'il n'y a un Aymara éternel, on ne saurait voir de l'ethnicité partout. (Wachtel 1992)
\end{abstract}

Choisir de questionner l'état des identités collectives andines sur les limites des États qui ont été fondés dans la région au XIX ${ }^{\mathrm{e}}$ siècle, c'est donc à la fois borner temporellement l'analyse et contextualiser un type de rapport au collectif. C'est aussi faire l'hypothèse d'une spécificité du rapport entre territoire et identité à la frontière dans la mesure où la ligne arbitraire qui est censée départager des groupes sociaux par leur assignation à un ensemble politique inédit n'a joué ce rôle que de façon imparfaite. Qu'est-ce donc qu'une frontière ? Dans son acception traditionnelle, la frontière signifie en effet la limite du champ de compétence d'un pouvoir : depuis l'invention de l'État-nation, celle-ci coïncide le plus souvent avec celle de l'extension de sa souveraineté. Nous considérons que cet objet spatial est tout autant une construction sociale qu'une institution, ce qui implique que les acteurs de la frontière soient aussi bien ceux qui la font ou la remettent en cause politiquement, à savoir les États, que tous ceux, qui, au quotidien, agissent autour et en fonction d'elle (Paasi 1998, 2009). On constate ainsi autour des frontières aussi bien la permanence de liens d'échange, préexistants à la séparation, que la stimulation de relations liées à l' " effetfrontière », c'est-à-dire encouragées par le différentiel entre les deux côtés de la ligne. La frontière marque ainsi le point de différenciation d'avec l'autre, elle matérialise l'altérité : toute frontière introduit « de la distance dans la proximité » (Arbaret-Schulz 2002). À ce titre, la frontière participe du processus de conformation/différenciation que l'on dit souvent caractériser l'identité, mais pas de façon aussi évidente qu'une carte politique où les États sont indiqués par des couleurs tranchées le laisserait entendre. 
L'évolution récente des frontières, leur ouverture dans la mondialisation, ne signifie en aucun cas le retour à l'état territorial antérieur ! Plutôt que de constater que la multiplication des échanges amène le monde à se passer des frontières, on observe que leur évolution ouvre un vaste chantier territorial qui bouscule les niveaux d'analyse, amène à retravailler la question des échelles territoriales et questionne les identités locales et nationales. L'examen des processus de recomposition frontalière et de construction transfrontalière peuvent se faire selon trois focales différentes (Amilhat Szary et Fourny 2006). On peut les comprendre d'abord selon leurs « structurations », en se fondant sur l'analyse des formes de constructions institutionnelles internationales : celles-ci sont nombreuses, malgré les difficultés de coopération dues aux discontinuités politiques, qui engendrent des régimes de gouvernance frontalière complexes, multiscalaires, et parfois dissymétriques. Dans le cas qui nous intéresse, on observe des liens inédits entre des municipalités rurales et des institutions internationales, qui bousculent le rapport à l'État des habitants des périphéries andines considérées. La deuxième lentille d'interprétation est celle des « franchissements » : la frontière apparaît dès lors comme une « ressource » qui engendre des flux et interactions constituant les territoires transfrontaliers. Il est intéressant d'observer que cette dynamique est loin d'être linéaire (dans bien des cas, des processus d'ouverture et de fermeture coexistent, $c f$. Amilhat Szary 2007), mais la mémoire du mouvement et de l'échange joue un rôle important dans les représentations de la frontière des populations limitrophes. La troisième focale est celle « identités » : les territoires transfrontaliers constituent des espaces identitaires spécifiques, capables d'incorporer au collectif l'altérité de l'outre frontière (à ce titre, bien des éléments mis en avant dans les deux étapes précédentes sont constitutifs de cette troisième focale). Ce sont ces trois composantes qui participent du processus de territorialisation des espaces qu'une frontière relie autant qu'elle les différencie, mais l'approche identitaire est sans doute celle qui permet de poser le mieux la question du rapport entre l'identité collective et individuelle.

Comment la transfrontaliarité se décline-t-elle dans des régions ou la notion de communauté contribue à façonner les identités de manière déterminante ? Nous tenterons de répondre par une approche depuis la périphérie de trois pays andins : ce point de vue nous paraît déterminant pour montrer que si chaque territoire est porteur de processus identitaires distincts, la contextualisation politique des transformations qui s'opèrent sur les frontières latino-américaines permet de dégager des tendances communes. Si l'identité collective repose traditionnellement sur le partage de normes et sur des « cadres collectifs de la mémoire » (Halbwachs 1950), on peut cependant considérer qu'elle dépend aussi du résultat d'une action partagée. Nous nous attacherons dans cette deuxième partie à la performativité de la frontière dans l'action collective engagée par des groupes ethniques sur les frontières du centre des Andes.

\section{Frontières d'État : héritage et échange}

\section{De l'ayllu à la nation, et retour}

Dans les Andes centrales, berceau pour beaucoup de l'idéal-type de ce que représente la « communauté » autochtone, le statut des identités collectives doit faire l'objet d'une lecture historique critique. La question y est d'autant plus sensible que la notion de communauté a été idéalisée à la fois à l'intérieur des systèmes politiques en place et par leurs détracteurs, 
selon des modalités variables depuis la colonisation. Dans un premier temps en effet, le «modèle » de la communauté indigène a été reconnu officiellement par la Couronne espagnole (au motif essentiel que les ressortissants de ces groupes étaient tenus de payer leur tribut directement à la Couronne, sans passer par les propriétaires terriens). Par la suite, et même si l'incorporation des Indiens dans les Républiques a été inégalitaire (notamment du fait des systèmes censitaires), la modalité communautaire n'a été que rarement supprimée du cadre légal de fonctionnement des États (Demélas 2003). Les descendants des premiers habitants des Andes ont bel et bien participé au façonnement des Républiques (dans le cadre de la transformation des rebellions contre la tutelle impériale), et les États nouvellement formés, sans reconnaître aux Indiens ces faits d'armes et le bénéfice politique qui pouvait en être attendu, n'ont pas nié ces communautés. Ils n'ont pourtant pas reconnu des droits civiques complets aux individus les constituant.

C'est dans la période de constitution des identités nationales, au moment où le rapport aux cultures précolombiennes s'est distendu, que le lien communautaire a changé de nature. Certains mouvements politiques ont pu alors idéaliser un passé précolonial fondé sur un ordre social dont la dimension collective fut relue au prisme socialiste (la communauté comme état premier du communisme, $c f$. Mariátegui 1968). Ces précisions nous invitent à éviter de considérer les périphéries andines comme des espaces échappant à l'autorité de l'État. M.D. Demélas nous rappelle que des « guerres comme celle du Pacifique [ont contribué] fortement à hybrider l'identité des habitants d'origine autochtone » (Demélas 2003) dans la mesure où elles amenèrent justement les populations des périphéries territoriales de l'État à se positionner par rapport au collectif. L'État pouvait alors apparaître comme un référent collectif nouveau, différent de la communauté plutôt du fait de son échelle que de sa nature. Toute une littérature récente travaille à démontrer que les périphéries constituent des lieux où lire l'État autrement, plutôt que des espaces à interpréter comme échappant par essence aux normes collectives. Le commerce frontalier par exemple ne constituerait par une menace pour l'intégrité territoriale : ces espaces longtemps brocardés pour l' " informalité » dangereuse des échanges qui s'y déroulaient constituent en fait des espaces-relais du pouvoir central (Bennafla 1999). Par contre, c'est sans doute dans les périodes de crise politique, que les identités de type ethnique ont été les plus vulnérables : au Chili, on a ainsi pu parler d' " holocauste au progrès » (Van Kessel 1980) pour qualifier la façon dont la dictature de Pinochet a réduit les populations aymaras du nord du pays au travers d'un découpage communal niant leur contour d'une part, et en niant leurs droits collectifs sur l'eau et la terre d'autre part.

La marginalisation territoriale des populations autochtones peut être lue à deux échelles : lors de la colonisation espagnole d'abord, les communautés ont été repoussées vers les terres les moins productives, les plus éloignées des centres urbains. Celles-ci s'avérant finalement les moins touchées par le métissage et l'assimilation politique. Par la suite, le tracé des frontières, fragmentant l'espace andin en différents États, a pu renforcer le caractère périphérique de la situation des populations autochtones, notamment en termes économiques (Karasik 1999 ; Benedetti et Argarañaz 2003). Mais l'imposition de lignes arbitraires séparant des pouvoirs centralisés a surtout eu pour effet de modifier les conditions des échanges traditionnels dans les régions considérées. La sédentarisation des populations andines sous la période coloniale est en effet demeurée incomplète tant que les circulations traditionnelles ont pu se maintenir entre les étages écologiques, et l'existence de caravanes est attestée 
jusque dans les années 1970 pour la zone que nous avons étudiée, entre la Bolivie, le Chili et l'Argentine (Nuñez Atencio et Dillehay 1995). La formalisation progressive du passage des frontières (contrôle régulier des papiers d'identité et des véhicules, réduction des points de traversée et de leur période d'ouverture) est finalement assez tardive (González Miranda 2000 ; Amilhat Szary 2007 ; González Miranda 2009), contemporaine de la présence de régimes militaires dans l'ensemble des pays concernés. La préoccupation de ces derniers pour la « sécurité intérieure » a justifié tant la mainmise sur les circulations transfrontalières que des découpages communaux inédits, sans respect des limites communautaires (au Chili, rassemblement en 1974 au sein d'une même commune de Los Cóndores de fractions de deux communautés d'Isluga et de Cariquima, leurs autres parties étant rattachées aux communes voisines de Huara, Chiapa et Camiña (Van Kessel 1980), achevant de déstructurer deux groupes dont les échanges avaient été déjà fort bousculés par le tracé de la frontière Bolivie/ Chili au cœur de leur territoire. Les critères normatifs de ces découpages modernes prenaient en effet appui sur des éléments caractéristiques du relief, notamment les volcans utilisés comme support aux limites politiques alors qu'ils constituent traditionnellement des repères identitaires centraux pour les communautés (lieu de vénération des ancêtres) (BouysseCassagne et Bouysse 1998).

C'est dans ce contexte de déstructuration du fonctionnement collectif des habitants autochtones des Andes centrales que s'est construit le mouvement de reconquête indigéniste. Cette période et ses mécanismes sont extrêmement bien documentés (Berdichewsky 1986 ; Bengoa 2000 ; Albo 2002 ; Gros 2003, 2007), renseignant la réappropriation progressive de leur culture par les populations autochtones et les revendications politiques l'accompagnant (concernant en tout premier lieu l'accès à la terre et la possibilité de la gestion communautaire des terres). Ces travaux montrent aussi l'ambiguïté du contenu identitaire des mouvements politiques qui revendiquent leur origine communautaire : celui-ci est formulé par des militants, vivant en ville, qui savent reconstruire un discours qui, devant être acceptable à la fois par les ressortissants des communautés et par leurs interlocuteurs, devient le produit de cette négociation permanente de la reconnaissance (Lamont et Bail 2005)1.

\section{Vers un décentrement de l'identité collective}

L'écho de ces revendications culturelles et l'émergence d'un mouvement politique fondé sur elles s'inscrivent dans un contexte où les modalités de l'action collective évoluent (Darnovsky, Epstein et Flacks 1995 ; Melucci 1996), permettant leur décentrement, c'est-àdire le fait que leurs initiateurs soient à la fois d'origine plus diversifiée et parlent au nom d'une multitude de groupes minoritaires (Holland, Foxt et Daro 2008). Il apparaît ainsi que l'engagement dans l'action participerait, autant que les composantes traditionnelles de l'appartenance, à la constitution de l'identité du groupe et de ses membres. On peut ainsi évoquer la " chronique d'une ethnie annoncée » à propos de la mobilisation d'une communauté villageoise des bords du salar d'Atacama, ayant perdu depuis plus d'un siècle sa langue et une grande partie de ses repères culturels d'autrefois, pour se construire un

1. H. Gundermann insiste d'ailleurs sur la différence entre processus d'identification et reconnaissance, selon qu'il différencie en fonction de l'analyse d'un préjugé de nature positive ou négative porté sur le groupe (Gundermann Kroll 2000). 
argumentaire politique qui réponde aux conditions ouvertes par la nouvelle Loi indigène du Chili (Barros 2004).

Il semble qu'au cours de cette période, on passe progressivement d'une définition de la communauté ou du collectif conçue en termes anthropologiques (fondée sur les conditions d'appartenance) à une approche plus sociologique (où les conditions de l'action jouent le rôle de critère distinctif). La projection socio-spatiale de ces conceptions se traduit par l'évolution des conditions de la territorialisation : on passerait d'une forme de territoire hérité (la portion de terre appropriée qui fonde l'identité historique) à un territoire fondé sur un projet (le « projet de territoire » produit par un jeu d'acteurs inédit sur une portion d'espace, $c f$. Gumuchian, Grasset, Lajarge et al. 2004). Cette tension entre les définitions du territoire ${ }^{2}$ permet d'éclairer ce qui se joue en ce moment sur une partie des frontières des Andes centrales, autour de l'identité aymara notamment.

L'inscription de ces revendications territoriales se comprend à la lumière de la reconstruction contemporaine des inter-relations entre le local et le global. Les communautés andines se caractérisaient en effet par un rapport traditionnel à l'espace fort différent du principe territorial européen : J. Murra (Murra et Wachtel 1978 ; Murra 1992) a utilisé le terme d'archipel pour qualifier l'organisation sociale dans les Andes, les groupes établissant généralement un noyau villageois où s'ancrait la communauté, mais vivant en lien permanent avec des établissements voisins, distants de plusieurs jours de marche en amont et en aval, pour multiplier leur accès aux ressources écologiques. La caractéristique de ces lieux périphériques était leur pluri-ethnicité, le fait qu'y vivaient ensemble des individus issus de différentes communautés et reliés à leurs centres respectifs par des pratiques de mobilité régulières. L'échange faisait alors partie intrinsèque du fonctionnement du groupe dont l'ancrage spatial était discontinu. Le tracé de limites de type moderne, pour des besoins administratifs et politiques, par les colons espagnols contredisait fondamentalement ces structures et imposait, par la sédentarisation, une transformation profonde des liens sociaux.

On a observé dans les Andes centrales, comme en Équateur (Massal 2005), une montée en puissance des mouvements indigénistes dont l'écho politique a atteint en une dizaine d'années un niveau national. Après l'institutionnalisation des partis indigènes, l'élection à la présidence de Bolivie d'Evo Morales (2006) est souvent considérée comme le symbole d'un certain aboutissement de cette dynamique. L'analyse de cette « reconquête » révèle cependant un certain brouillage des identités : les mouvements indigénistes boliviens sont nés dans les Basses Terres, et en Équateur, ce sont les Shuars amazoniens qui se sont mobilisés d'abord (Fontaine 2004 ; Lacroix 2007).

L'expression des revendications territoriales fondées sur l'argumentation autochtone n'intervient sur les frontières que dans une étape ultérieure, récente, témoignant sans doute, $a$ posteriori, de deux paradoxes : la force du sentiment national à la périphérie de l'État d'une part, la puissance de l'État dans la structuration du jeu politique d'autre part. « Dans un sens, les études d'organisations indigènes en Amérique latine ont fini par refléter ce qu'Agnew (1987³)

2. Pour plus de détails sur le débat épistémologique, $c f$. les différentes contributions qui font la rubrique « Territoires » dans Lévy et Lussault 2003.

3. Cité par Perreault 2003 : "In this sense, studies of indigenous organizations in Latin America have ended to mirror what Agnew (1987) identified as the "cult of the state", that is, the privileging of the state as the locus and scale of political practice". 
avait identifié comme le « culte de l'État », c'est-à-dire le fait de privilégier l'État comme le lieu et l'échelle des pratiques politiques » (Perreault 2003). En interrogeant les dynamiques local/global de façon comparative, aux frontières de trois pays, nous souhaitions creuser ce paradoxe d'une identité collective communautaire reformulée par l'intermédiation nationale.

Certains sont allés jusqu'à parler de « dérive populiste » des mouvements indigènes dans la mesure où leur argumentaire reprend à leur compte la sémantique nationaliste, moyennant adaptation des référents et des mythes fondateurs (Le Bot 2006). Cet argument ne nous paraît recevable que dans la mesure où l'on se réfère à une définition discursive du populisme (Joffres 2008), conçu comme un style politique "susceptible de mettre en forme divers matériaux symboliques et de se fixer en de multiples lieux idéologiques, prenant la coloration politique du lieu d'accueil » (Taguieff 2002). Mobiliser cette notion permet ainsi d'insister sur « l'exploitation systématique du rêve ", « rêve d'immédiateté, de proximité, de contact direct, [...] Ce qui indique l'importance du mythique dans le populisme, qui relève ainsi de l'anthropologie de l'imaginaire sociopolitique moderne » (Hermet 2001). C'est pour discuter du rapport à l'imaginaire collectif des projets politiques menés en zone frontalière, par des populations d'origine aymara, qu'il nous a semblé intéressant de mobiliser une grille de lecture idéologique.

\section{Frontières globalisées : idéologie et innovation}

Aux frontières des Andes centrales, l'innovation territoriale?

La région que nous avons choisi d'examiner, aux frontières du Pérou, Chili et de Bolivie, se caractérise par l'émergence récente d'une structuration politique internationale, autour de l'identité aymara (Amilhat Szary et Rouvière 2010 ; González Miranda, Rouvière et Ovando 2008 ; Amilhat Szary 2009). Les Aymaras constituent un groupe présent sur ces trois États de façon très inégale (1 250000 en Bolivie, 300000 au Pérou et 50000 au Chili ; Albo 2000). Leur répartition dans la zone frontalière analysée ne suit que partiellement leur distribution nationale : ils sont certes plus importants dans le sud bolivien (140 000), mais relativement peu nombreux au sud Pérou (18 000) par rapport au nord Chili (23000 - chiffres tirés des derniers recensements des trois pays pour les communes rurales concernées, sachant que la discrimination ethnique repose sur une auto-identification identitaire).

À la triple frontière entre le Chili, le Pérou et la Bolivie nous avons observé, depuis son origine à la fin des années 1990, un regroupement politique original entre des maires ruraux promouvant leur identité ethnique pour tisser des liens transfrontaliers, dans l'optique d'œuvrer ensemble pour le développement de leurs territoires. L'initiative repose à la fois sur la promotion de la production agricole locale (quinoa et origan) et sur le développement de ressources nouvelles (tourisme et aménagements d'infrastructures routières). Son originalité tient en grande partie au lieu où elle émerge, plus connu pour sa conflictualité géopolitique que pour ce type de politiques intégratrices. On se trouve en effet sur des frontières tracées à l'issue d'un conflit qui a conditionné sur le long terme les relations diplomatiques entre les trois pays. La guerre du Pacifique (1879-1884) permit effectivement au Chili de conquérir la région péruvienne de Tarapaca ainsi que la région d'Antofagasta sur la Bolivie, privant ainsi ce pays d'accès à la mer. L'espace de Tarapacá est d'un intérêt stratégique tout particulier : il est l'un des fondements des mémoires collectives nationales chilienne et péruvienne, mais fut aussi au cours du $\mathrm{XX}^{\mathrm{e}}$ siècle l'objet de vaines et récurrentes négociations entre Chili 
et Bolivie pour la sortie maritime de celle-ci sur le port d'Arica. La contestation de ces limites a longtemps été le principal objet de disputes ${ }^{4}$ relayées par une "guerre de papier» permanente entre les trois pays (Gonzalez Miranda 2006 : 111). La victoire du Chili a aussi signifié pour ce pays l'apparition d'une population aymara ${ }^{5}$, antérieurement péruvienne, sur ses marges septentrionales. La mobilisation d'une mémoire collective subrégionale apparaît donc d'emblée contradictoire du fait des conflits nationaux; elle rivalise avec un argument ethnique fondé sur une réalité précolombienne intégratrice (Albo, Arratia, Hidalgo et al. 1996). Depuis l'établissement de la carte politique à l'issue de la guerre du Pacifique, même si les échanges familiaux et régionaux ont continué à traverser cet espace, nourris à la fois par des flux commerciaux de produits agricoles et par des migrations de travail (González Cortes et Gundermann Kroll 1994 ; González Cortez 1995), l'identité politique s'est effet construite dans une perspective nationale.

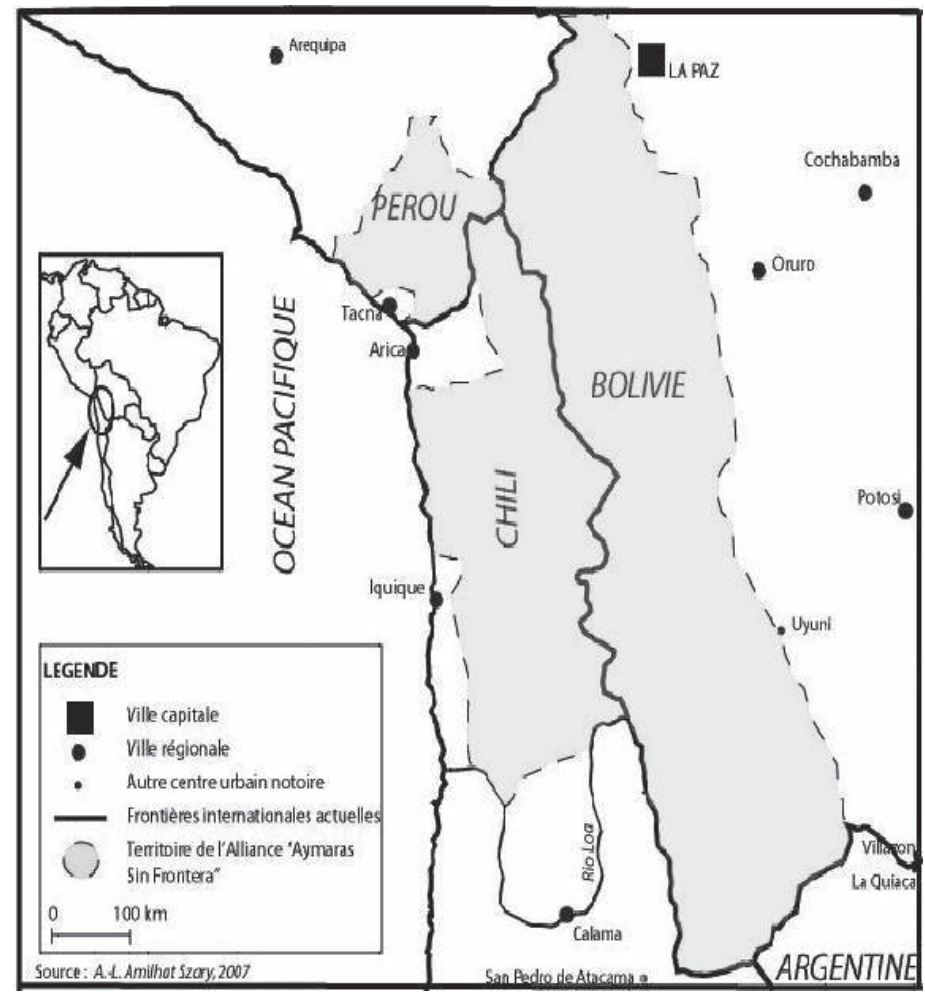

Figure 1

Carte de situation de l'Allliance Stratégique « Aymaras Sin Fronteras ».

4. Traité de Lima de 1929 appliqué en 1999 seulement, rupture diplomatique entre Chili et Bolivie en 1978.

5. On considère aujourd'hui que la population aymara est le troisième groupe originaire du continent après les Quechuas et les Guaranis. Environ 2 millions d'individus parlant aymara se répartissent pour 1,7 millions d'entre eux en Bolivie, 500000 au Pérou et 20000 au Chili. (cf. Albo 2000 : 43-73 ; Albo 2000 : 44). 
Le paradoxe de cet exemple est bien que le leadership soit ancré dans celui des trois pays où les Aymaras sont les moins représentés. C'est également là où leur définition comme minorité fait le moins question : les identités ethniques sont peu présentes au Chili, et parmi elles la question aymara occupe beaucoup moins le terrain du débat politique que la question mapuche. La situation est inversée en Bolivie où non seulement les Aymaras constituent le groupe ethnique majoritaire, avec un président les représentant, mais où le territoire frontalier est beaucoup moins périphérique : si l'on se base sur une définition large du territoire couvert par l'Alliance comme recouvrant les régions frontalières, le département de La Paz, et la ville capitale elle-même devraient en faire partie. C'est au Pérou que la situation est la plus ambivalente : les Aymaras sont un des grands groupes ethniques, mais l'identité quechua est plus volontiers mobilisée dans la réappropriation de l'histoire nationale ; la décentralisation y a connu un développement très irrégulier qui rend la prise d'initiative locale encore très hésitante. Le statut du référent ethnique est donc très distinct dans les trois pays.

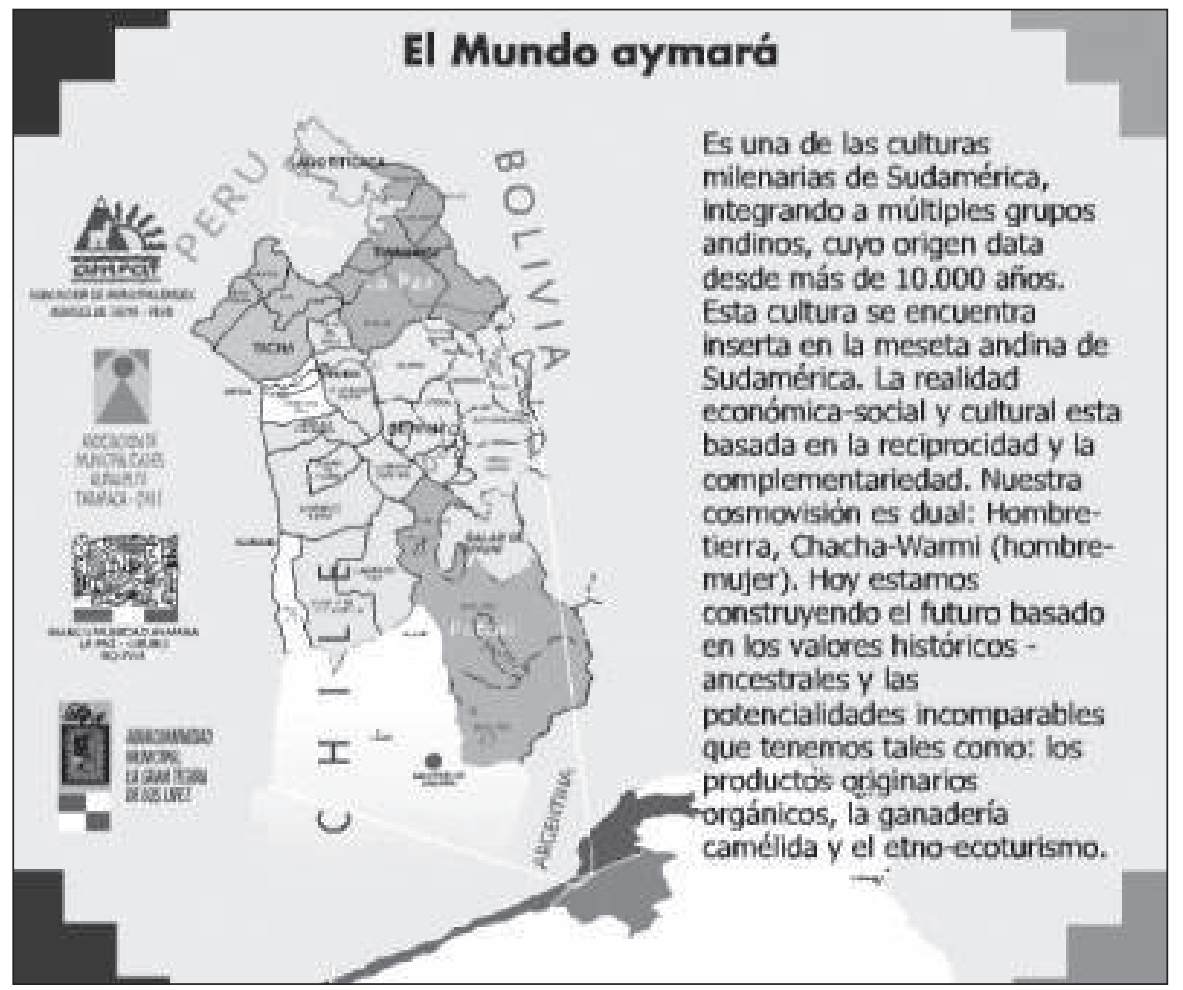

Figure 2

Carte tirée du site web de l'Alliance :

les territoires administratifs contemporains sont expliqués par la mobilisation de l'histoire précolombienne (2005). 
Aussi, quand des maires ruraux à la tête de cinq associations municipales préexistantes sur les territoires nationaux ${ }^{6}$, rassemblant au total cinquante-six communes, décidèrent de se rapprocher pour construire ensemble un projet politique, ils faisaient œuvre inédite. Ils aboutirent à la création en 2001 d'une "Alliance Stratégique Aymaras Sans Frontières ». Ce regroupement fut possible grâce à l'investissement personnel du maire d'un village de la province de Parinacota (Chili), lié d'amitié avec quelques maires péruviens et boliviens. L'actuel président de l'Alliance Stratégique et maire de Putre (commune de l'arrière-pays d'Arica au Chili), Francisco Humire lie sa création à l'existence dans sa commune d'une foire andine (la FERAN, Feria andina) initiée en 1997, qui aurait joué le rôle de point de rencontre entre les acteurs concernés dans les trois pays. L'enjeu pour lui était double : prendre la tête de l'association des maires ruraux du nord chilien grâce à un projet qui échappait à son rival qui la présidait alors, mais aussi se construire une carrière politique internationale. Cette configuration ne suffit pas tout à fait à expliquer pourquoi le leadership du mouvement est chilien alors que c'est dans ce pays que les Aymaras sont les moins nombreux.

L'écho rencontré par l'initiative dans les pays voisins a reposé sur la communauté d'intérêts, identifiée par les maires, de territoires ruraux marginalisés chacun dans leur pays du fait de leur situation périphérique, mais aussi de leur caractère ethnique (sauf en Bolivie où l'ethnie est majoritaire). Il faut donc se pencher sur l'argumentaire construit dans les trois pays pour soutenir une initiative conjointe. Si l'on s'attache à l'analyse des objectifs de Aymaras Sin Fronteras tels qu'ils sont affichés dans les documents produits par l'Alliance elle-même, on perçoit deux préoccupations distinctes : mener à bien des projets de développement permettant d'améliorer le niveau de vie sur l'espace concerné (électrification rurale, améliorations agricoles et pastorales, construction d'un projet de tourisme durable dans le cadre de la patrimonialisation des Chemins de l'Inca, etc.) mais surtout, semble-til, médiatiser ce territoire pauvre, éloigné, oublié, aussi bien auprès des gouvernements et bailleurs que des médias : "Nous devons faire connaître nos réalités » statue le premier texte (Aymaras sin Fronteras, Actes de l'accord de Putre, 2001), afin de « remporter l'appui des gouvernements régionaux, nationaux et des sources de coopération » (Aymaras sin Fronteras, memorandum d'Arica, janvier 2002) ${ }^{7}$.

C'est dans ce but que le regroupement transfrontalier est né, selon un raisonnement économique de gains de moyens : il s'agissait de « tirer profit des avantages et potentialités de l'intégration transfrontalière pour obtenir des bénéfices communs lesquels généreront à leur tour des externalités positives dans leur entourage, améliorant les niveaux de subsistance » (Aymaras sin Fronteras, memorandum d'Arica, janvier 2002). L'alliance ne se présentait pourtant pas comme une association de nature productive : les acteurs réunis parlaient plutôt de la création d'un cadre commun de réflexion et de coordination des initiatives d'interaction et de développement transfrontalier, ainsi qu'en témoigne ce long extrait de la Déclaration de Tacna (août 2001).

6. Il s'agit de l'association des municipalités rurales de Tarapaca (Chili), l'association des municipalités rurales andines de Tacna (AMRAT, Pérou) et des trois Mancomunidades de la Paz, Oruro et Lipez-Potosi (Bolivie).

7. « Lograr el aproyo de los gobiernos regionales, nacionales y de las fuentes cooperantes », Plan trinational de développement transfrontalier, 2002. 


\begin{abstract}
Attendu que :
- les espaces Régionaux Frontaliers constituent des unités économiques et sociales aux potentialités de développement commun et partagé nombreuses et fortes ${ }^{8}$,

- le processus de globalisation, comme les facteurs de compétitivité qui s'imposent, ont pulvérisé les formes artisanales et traditionnelles de notre système de production et de commercialisation,

- dans le cadre des signaux clairs en faveur des initiatives de développement dans les zones pauvres donnés par des Organismes Multilatéraux, il existe une volonté politique concertée de la part des protagonistes de cette rencontre,

- les ressources de cette région sud-andine sont similaires dans tous les pays concernés, puisque des réalités identiques nous unissent, comme l'environnement, la culture et le développement productif,

- nous devons faire connaître nos réalités.
\end{abstract}

Il semble que les contacts avec l'extérieur ont ensuite grandement stimulé l'essor de l'Alliance : ce qui donne à cette expérience lointaine une résonance étonnante, c'est en effet la rencontre de ces maires montagnards avec des fonctionnaires internationaux de la Banque Mondiale et leur capacité à tirer parti de la convergence d'intérêts, ou du moins de discours et de référents, entre eux. Cette coïncidence est liée à l'existence au sein de la Banque d'une division de projets très attentive au développement des minorités, qui a su tirer parti de l'information du projet de l'Alliance, cette nouvelle n'ayant pas paru véritablement digne d'intérêt au niveau des instances nationales des pays concernés.

Relayés par des ONG locales dédiées au développement des côtés péruviens et boliviens, les maires réussirent à obtenir, dès la fin de l'année 2001 à la Banque Mondiale (BM), le financement de trois réunions de concertation jusqu'en 2003, sans que celle-ci puisse aller plus loin pour assurer le suivi du projet sur le moyen terme, faute d'assise institutionnelle de l'Alliance. Pourtant ces rencontres et le travail conjoint des maires et des fonctionnaires de la BM permirent d'un côté de faire connaître cette expérience marginale à Santiago, de l'autre d'approfondir les orientations du projet. Dans un premier temps, il s'agissait de lancer un projet de tourisme rural intégré, puis une deuxième réunion à Arica en 2002 a donné lieu à un document proposé par les agents de la $\mathrm{BM}$, intitulé « Une proposition d'empowerment pour les Aymaras $»^{9}$. Les trois axes principaux de ce document portaient sur la formation des membres de l'alliance au développement, leur connaissance des agents de développement externe et le renforcement du cadre légal de l'Alliance. Au terme de cette première phase de coopération, le sous-secrétariat de développement régional (SUBDERE), dépendant du ministère de l'intérieur chilien, s'engagea à porter un appui technique au projet, et en 2006 un consultant de Santiago fut délégué à la région de Tarapacá pour une période d'un an : c'est lui qui monta un dossier auprès de la Banque Interaméricaine de Développement (BID), soumettant la candidature de ce qui était en train de devenir un projet de territoire au concours de « Bien Public Régional », devant impliquer au moins trois pays dans un projet commun. Il lui revient d'avoir reformulé le projet de l'Alliance selon les codes intelligibles par la BID, cela au nom de la région de Tarapacá - et non pas de la SUBDERE - afin de faire preuve du

8. «Amplios y poderosos factores de desarrollo conjunto y compartido ».

9. Voir le rapport du séminaire en ligne : http://wbln0018.worldbank.org/LAC/lacinfoclient.nsf/6f1c77f445ed aa6585256746007718fe/61f37aa3d9fa68b585256d9d00611468/\$FILE/SeminarioAymaras.pdf 
caractère « décentralisé » du projet. C'est en fait le dépôt de ce projet auprès de la BID qui a légitimé la coopération entre les régions frontalières, une « Convention aymara » étant signée entre les régions et départements concernés.

Présenté sous le titre « Récupération, développement et mise en valeur du patrimoine culturel et naturel aymara », le projet fut accepté par la BID en 2007 sous le nom simplifié de « Héritage naturel et culturel aymara ». Cette étape de légitimation internationale s'est révélée essentielle pour éveiller l'intérêt des maires dits "passifs », ce notamment du côté péruvien. L'initiative locale est donc à nuancer dans ce cas : elle concerne quelques entrepreneurs politiques dont le succès auprès des autres maires « aymaras » dépend de leur capacité à attirer des fonds vers les espaces locaux. Si les objectifs de l'Alliance ne sont pas encore concrétisés en termes d'infrastructures, le suivi des étapes de sa consolidation permet d'observer d'un côté comment les paradigmes internationaux du développement sont concrètement réappropriés dans les espaces locaux frontaliers, de l'autre comment l'intervention de la coopération internationale détermine les conditions de pérennité d'un projet territorial transfrontalier. Elle permet aussi de comprendre comment l'ethnicité se négocie dans la proximité culturelle historique et la différenciation politique engendrée par la présence de la frontière.

Dans un premier temps, les acteurs concernés se revendiquaient surtout de leur ruralité : et au moment de sa première Convention, l'Alliance ne s'appelait encore que «Alianza Estrategica Trinacional de Alcaldes Rurales Andinos de Bolivia, Chile, Perú » (mars 2002), et c'est au titre d'une rencontre (« encuentro ») de maires « andins » de Bolivie, du Chili et du Pérou qu'elle fut célébrée dans la presse locale. Le document co-produit avec la Banque Mondiale semble révélateur d'un changement de paradigme dans la revendication : le texte se présente comme une "stratégie sur les municipalités indigènes : proposition pour un renforcement du pouvoir aymara » («La estrategia sobre municipalidades indigenas : una propuesta de empoderamiento para los Aymaras »). Il donne à l'Alliance un triple rôle, le pilier identitaire apparaissant essentiel («1- Construire un espace social pour appuyer la récupération de son identité ; 2- Mobiliser sa capacité institutionnelle ; 3- Fortifier ses capacités de gestion dans la définition et la promotion de stratégies de développement rural ». Le bailleur ne fait que mettre en valeur un discours porté par certains membres de l'Alliance, notamment le président de la FERAN F. Humire, dont un certain nombre de courriers attestent de l'intensité de son intériorisation de la rhétorique ethnique. Il parle souvent de « l'espoir ressenti si anxieusement par certains de ses membres d'une intégration des communautés de ce territoire aymara, ancestralement commun dans cette zone frontalière » $(2001)^{10}$, et s'en sert comme d'un argument devant valider la maturation du projet stratégique : « Ce document, est le produit d'un travail silencieux et concentré, comme un apport additionnel à l'aspiration intense à l'Intégration [la majuscule est de l'auteur] de ces trois pays concernés. Ces derniers désirent fortifier et développer un territoire régional frontalier, dans lequel sont impliqués des hommes et des femmes dont la seule motivation est d'avoir l'opportunité de se sentir appartenir à cette zone sud-andine, dont le dénominateur commun est d'être originaires et fils de cette terre-mère, orgueilleux de leur culture et de leur origine aymara »

10. Lettre à un consultant, 2001 (PHDG/Asesor Consultor ) « Tan ansiada integración de los componentes de las comunidades de este territorio aymara, ancestralmente común para esta zona fronteriza ».. 
$(2002)^{11}$. Cette rhétorique lui permet d'emprunter des accents provocateurs dans sa façon de dénoncer l' " amnésie » des gouvernements centraux dans leur façon de répondre aux besoins des communautés rurales andines » et d'affirmer que « nous ne voulons pas que nos communautés se convertissent en un second Chiapas », suggérant que l'Alliance est capable de « produire un pôle de développement dans toute la macro-région du monde aymara $»^{12}$.

La sémiologie des documents de communication produits par ASF témoigne de cette évolution ( $c f$. figure 2) : si le logo tri-frontalier (trois mains prolongées d'un drapeau qui se serrent) reste un marqueur stable, les référents au renouveau identitaire andin sont fortement présents, avec l'insertion notable de la Wiphala, le drapeau à petits carreaux multicolores qui est devenu la bannière des mouvements politiques indigénistes en Bolivie notamment. Vieilles pierres de Tiwanaku, textiles anciens et danses folkloriques constituent des attributs plus facilement identifiables par des non-Aymaras et participent de l'effort de l'Alliance pour utiliser la culture dans un but productif.

La médiation d'un organisme international dans la formulation de l'identité aymara doit être questionnée. Cet exemple conforte une dynamique de promotion d'identités transnationales qui s'appuie sur le renouvellement des paradigmes internationaux du développement. Il illustre a posteriori l'influence d'institutions dont le rôle est souvent minimisé par les acteurs de terrain, et que l'on peut définir comme " panethniques », dont les plus célèbres restent à ce jour la Conférence Inuit Circumpolaire (fondée en 1977 en Alaska, reconnue en 1982 par l'ONU comme ONG), la COICA (1984, Coordination des organisations indigènes du Bassin amazonien ${ }^{13}$ ), elle-même membre fondateur de l'Alliance des peuples autochtones des forêts tropicales d'Amérique centrale, d'Amérique du Sud, d'Asie et d'Océanie en 1992. Mais si l'apport financier permet évidemment de comprendre la mobilisation des acteurs dans tous les pays concernés, il ne peut à lui seul expliquer le succès de la mobilisation identitaire. Il semble que ce soit bel et bien la situation frontalière qui ait permis cette construction politique inédite.

11. Lettre du 28/12/01 de Humire à F. Vidal V., secrétaire d'État à l'Aménagement (SUBDERE), sur le document d'accord signé à Putre Feran 2001 : c'est « Este documento, es el producto de un trabajo silencioso y dedicado como un aporte más a la tan anhelada Integración de estos tres países involucrados. Quienes desean fortalecer y desarrollar un territorio regional fronterizo, en donde están involucrados hombres y mujeres que solo los animan a tener una oportunidad de sentirse parte de esta zona sur Andina, con un denominador común el cual es ser originarios e hijos de esta madre tierra, orgullosos de su Cultura y de su origen Aymará. »

12. Déclarations à la presse de F. Humire récemment élu président de l'ASF (El Correo, Tacna, 23 et 24/3/2002).

13. Elle-même issue de dynamiques ayant d'abord pris leur essor à l'échelle nationale, comme les fédérations concurrentes organisées à l'échelle du Pérou : l'AIDESEP, Asociación Interétnica de Desarrollo para la Selva Peruana et la CONAP, Confederación de Nacionalidades de la Amazonía Peruana. 


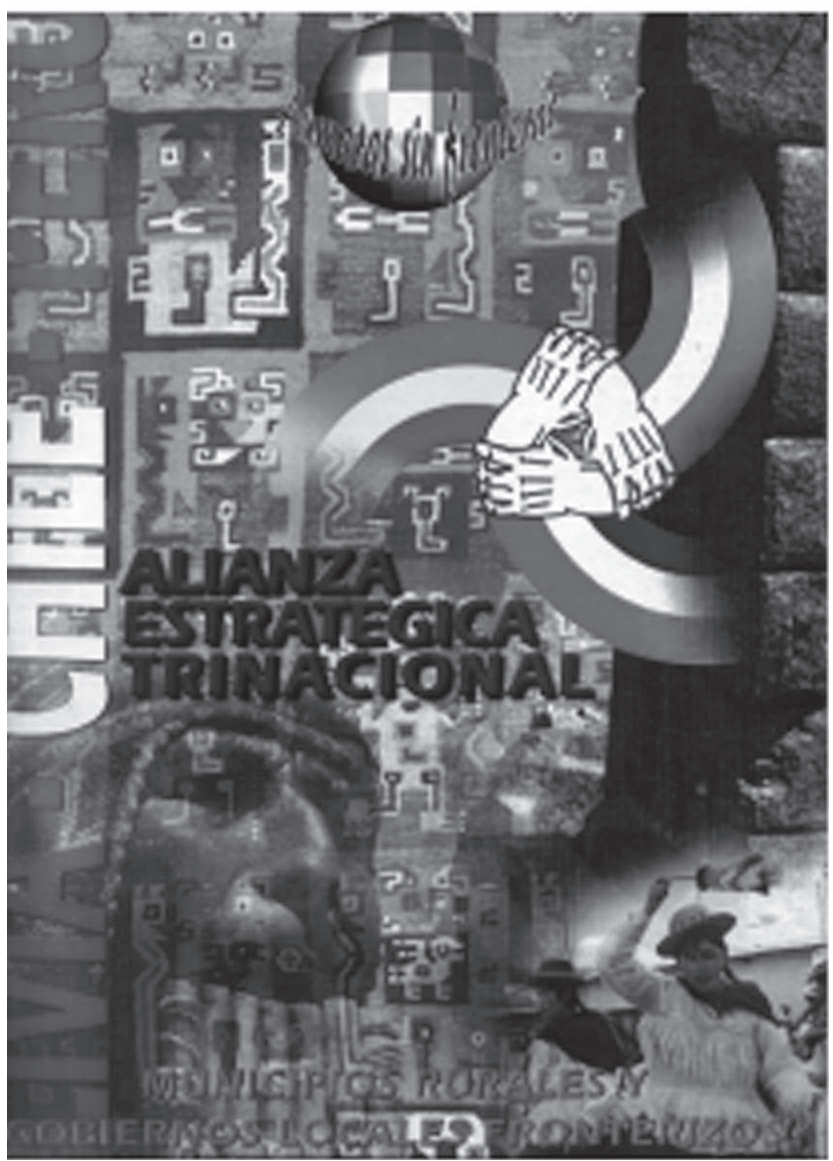

Figure 3

Couverture de pochette réalisée pour rencontres trinationales, 2002.

La frontière internationale représente ici un double référent symbolique, en tant qu'elle représente l'autorité de l'État. On cherche d'abord à la dépasser pour provoquer la réaction de l'institution, espérant que cette dernière se traduira par une volonté d'investissement public. Mais il faut aussi se démarquer de la nation que ce cadre induit : se définir par son appartenance ethnique, qui plus est en définissant un territoire indigène, c'est se donner d'autres frontières anthropologiques et politiques. La situation frontalière permettrait ainsi de décupler l'intensité d'une construction identitaire (Eskelinen, Liikanen et al. 1999) mais aussi de rendre plus visible un projet de territoire (Newman et Paasi 1998). Du point de vue de l'État, de tels projets peuvent présenter l'avantage d'une certaine souplesse politique : ils laissent la possibilité de négocier les termes d'une reconnaissance territoriale sans menacer l'intégrité du pays par des dérives sécessionnistes (Gros 2003 ; Gros et Strigler 2006). Cela explique sans doute pourquoi un tel projet est né dans un contexte structurellement conflictuel où la légitimité des élites locales à aller contre les orientations des gouvernements centraux 
pouvait apparaître très faible. Les rapprochements diplomatiques récents des pays concernés (dans le cadre des blocs d'intégration macro-régionale, avec notamment l'entrée du Chili dans la CAN en 2006, mais aussi pendant la période où M. Bachelet et d'E. Morales ont gouverné leurs pays respectifs et ouvert le dialogue) ouvraient de nouvelles fenêtres d'opportunité aux maires frontaliers, mais l'expérience de l'AS ne s'inscrivit pas dans ce cadre. Il s'est plutôt agi d'une initiative parallèle, complétant ou contrebalançant ces dynamiques tout en relevant d'autres logiques de légitimation, plus diffuses et peu institutionnalisées. La situation géographique de l'Alliance a donc permis à ses promoteurs d'élaborer une stratégie de mise en visibilité, qui n'était pas seulement dirigée vers le niveau national ou régional mais aussi vers le niveau international.

\section{Déclinaisons idéologiques de l'identité}

La reconnaissance par des institutions extérieures n'en joue pas moins un rôle essentiel, dans la structuration de l'Alliance Stratégique. Dans ce processus, on a assisté à l' « incorporation asymétrique de groupes structuralement dissemblables dans une même économie politique », ce qui constitue pour certain une nouvelle définition de l'ethnicité (Lozano B. 2000 : 161). Le texte de N. Wachtel pris comme appui de notre réflexion se concluait en ouvrant le débat sur le statut du religieux dans la constitution des modalités d'appartenance, on peut s'y référer pour comprendre les référents identitaires dans un sens plus large : «Entre le rejet du passé et la foi du souvenir, comment se partage leur mémoire ? Dans quelle mesure les compromis, les syncrétismes qu'ils élaborent eux aussi, versent-ils dans des glissements incontrôlés au terme desquels ne se maintient qu'une identité illusoire? Et cette illusion, vécue avec un reste de ferveur, ne comporterait-elle pas une part de vérité ? (Wachtel, ibid.). L'emploi même $\mathrm{du}$ terme de syncrétisme est aujourd'hui remis en question pour montrer que les différents composants de l'identité ne s'assimilent pas, que les individus peuvent être porteurs de référents complexes et parfois contradictoires. Qu'en est-il alors des territoires ? Dans ce cas étudié, les différences culturelles ne représentent plus un produit résiduel de l'histoire mais fonctionnent comme une coproduction de facteurs actifs de la globalisation.

On comprend mieux dès lors que tout discours identitaire unifiant doit être lu au titre de sa force symbolique tout autant qu'à celui de ses référents culturels historiques. C'est à ce titre que l'on peut se demander si l'appartenance territoriale collective, du moins telle qu'elle est promue dans des projets de territoire comme celui que nous avons détaillé, ne serait pas de nature idéologique. L'utilisation de ce terme nécessite des précautions rhétoriques de même nature que celui du recours à l'idée populiste. Le mot recouvre en effet tout à la fois l'idée de légitimation du processus social et sa dimension symbolique.

L'une des premières incursions de la géographie sur ce domaine avançait que " l'idéologie peut être interprétée comme ce qui donne au groupe conscience de son être. Le thème d'identification est variable d'une culture à l'autre : cela peut être l'appartenance à une certaine lignée généalogique, le genre de vie que l'on pratique, le territoire où l'on vit en commun ou bien le partage de certaines valeurs » (Claval 1985). De façon générale, on a montré plus récemment que la notion de territoire elle-même était liée à un contexte idéologique, celui de l'établissement de la forme étatique dans l'Europe moderne (Elden 2010). On peut aisément étendre ce raisonnement aux frontières, établies comme corollaire des territoires nationaux et pour les borner. Il ne s'agit pas ici de disqualifier toute analyse territoriale au prisme d'un discours post-moderne trop large. 
Nous proposons se suivre en revanche les pistes ouvertes par P. Ricoeur (1986) pour éclairer notre compréhension des mécanismes de l'identité territoriale sur les marges. Sur la base d'une analyse renouvelée des représentations, il proposait de ne pas s'en tenir à une réduction de l'idéologie à un discours de distorsion. Au contraire, il mettait en avant un modèle motivationnel : pour lui la croyance devait être permise, car elle seule permettait de comprendre la fonction de légitimation du pouvoir politique (et d'expliquer le décalage entre les concepts wébériens de la revendication d'une autorité et celui de la légitimité de l'État). À ce titre, la dimension symbolique du discours devait être reconnue, et valorisée du fait de la fonction intégratrice, tant socialement que politiquement. L'idéologie définie comme une médiation symbolique devenait dès lors une déclinaison positive du discours identitaire dans le processus social. On comprend sans doute mieux ainsi comment l'autorité peut être légitimée par la culture, et de quelle façon la promotion d'un discours culturel permet de construire un processus politique. L'Alliance Stratégique apparaît dès lors comme un exemple intéressant pour mettre en lumière la dimension symbolique des identités collectives contemporaines, et leur capacité médiatrice à produire de l'innovation politique.

\section{Conclusion}

Dans la vision politique du territoire qui est présentée, la périphéricité est revendiquée aujourd'hui de façon originale : jusqu'alors analysée du point de vue de ses conséquences économiques et sociales, elle peut constituer le cœur de stratégies territoriales (Grimson 1999). On peut y lire une transformation importante du rapport de l'État au territoire, le modèle corporatiste étant révolu, l'État néolibéral est aujourd'hui le produit d'une mosaïque territoriale complexe au sein de laquelle l'indigène ne peut plus être assigné à catégorie sociale ou spatiale. Après de nombreuses recherches sur la communalisation des intérêts ethniques (Radcliffe, Laurie et Andolina 2002 ; Gundermann Kroll 2003), la question se pose de sa présidentialisation, qui transforme l'indigénisme en nouveau nationalisme, en Équateur ou au Pérou. Les recherches sur les confins frontaliers permettent de questionner autrement le lien entre souveraineté et identité, de revenir sur l'opposition essentielle construite entre l'autre et le même : ils s'y trouvent en interaction constante. L'analyse de la globalisation des enjeux politiques pour les populations indigènes permet alors de comprendre le renouveau de l'expression territoriale de l'ethnicité qui passe par les formes d'innovation décrites dans cet article.

L'examen de l'évolution des référents identitaires dans des territoires transfrontaliers de trois pays andins permet de définir les modalités de territorialisation qui y sont en cours. On voit combien les processus d'identification au territoire ont évolué avec la sédentarisation progressive (à la fois à l'échelle communautaire et nationale) de groupes sociaux habitués à des pratiques spatiales discontinues. Il apparaît cependant que ces processus de conformation/ différenciation y évoluent rapidement dans des contextes où l'appartenance et les liens sociaux ne sont que partiellement territorialisés. Dès lors que d'autres possibilités de la reconnaissance du groupe surgissent, elles sont rapidement mobilisées.

Les innovations observées peuvent être interprétées à lumière de la grille de F. Giraut (2009) : sous couvert de recherche de développement (amélioration des qualités du milieu), l'initiative Aymaras Sin Fronteras semblait construite sur la base de la rénovation du rapport centre/périphérie... Or on assiste bien ici à des avancées en " matière d'organisation et d'arrangements territoriaux (...) approche qui (...) s'intéresse aux formes innovantes de 
constructions spatiales et institutionnelles qui dépassent les codes étroits de la modernité territoriale et de ses pavages : souveraineté exclusive, continuité, limites fixes, emboîtement strict... ». Dans le contexte contemporain, les circulations culturelles (mots d'ordre et références globalisées) font effet localement ; elles prennent d'autant mieux dans les lieux périphériques comme les frontières, où l'habitude du jeu différentiel entre les États a construit une tradition de négociation pluri-institutionnelle.

L'ouverture des acteurs en présence vers la dimension transnationale (Santana 2003) semble avoir considérablement participé au renforcement du discours identitaire. La reconnaissance extérieure joue en effet de façon essentielle dans le devenir du groupe, et d'autant plus que cette dernière est institutionnalisée. Mais ce qui est en jeu est à la fois anthropologique et politique : les débats que nous avons retracés ici ne sont pas suivis par l'ensemble des habitants des communautés concernées. Dans le cas de l'Alliance Aymaras Sans Frontières, certains des leaders les plus actifs ont été dénoncés par leurs électeurs pour mauvais usage des fonds publics : l'international n'apparaissant pas comme une compétence a priori des maires d'une part, les bénéfices des fonds internationaux octroyés mettant un temps important à se concrétiser pour les habitants, étant consacrés dans un premier temps exclusivement des études de faisabilité et des plans stratégiques. Pour autant, le statut des territoires administratifs concernés a considérablement évolué pendant la période considérée, et le rapport avec les pouvoirs centraux des trois États a évolué en faveur des périphéries. Difficile de conclure sur un processus en cours dont les résultats sont encore difficiles à mesurer au niveau individuel : le cas étudié permet de mettre en lumière la complexité de l'analyse de discours et sa dimension symbolique, et par là de contribuer à renouveler un contexte épistémologique où les interactions entre discours culturels et politiques sont encore peu saisies en tant que telles.

\section{Références citées}

Albo, X., 2000. Aymaras entre Bolivia, Perú y Chile. Estudios Atacameños 19 : 43-73.

----, 2002. Pueblos indios en la politica. La Paz : CIPCA.

Albo, X., M. I. Arratia, J. Hidalgo et al. (dir.), 1996. La integración surandina cinco siglos después. CuzcoArica-Antofagasta : Centro Bartolomé de Las Casas/Corporación Norte Grande/Universidad Católica del Norte.

Agnew, J., 1987. Place and politics: The geographical mediation of state and society. Winchester/Boston : Allen \& Unwin.

Amilhat Szary, A.-L., 2007. « Are borders more easily crossed today ? The paradox of contemporary transborder mobilities in the Andes », Geopolitics 2007 (1) : 1-18.

2009. «Ruralité, ethnicité et montagne : Le référent andin dans le projet de territoire "aymaras sin frontera" - Rurality, ethnicity and mountain areas : The Andean referent in the "Aymaras sin Fronteras" project ", Revue de Géographie Alpine - Journal of Alpine Research 97 (2) (Les régions de montagne comme référents de l'action collective), http://rga.revues.org/index913.html (version française); http://rga. revues.org/index 933.html (version anglaise).

Amilhat Szary, A.-L. et M.-C. Fourny (dir.), 2006. Après les frontières, avec la frontière. Nouvelles dynamiques transfrontalières en Europe. La Tour d'Aigues : Éditions de l'Aube.

Amilhat Szary, A.-L. et L. Rouvière, 2010 [2007]. « Des dynamiques transfrontalières au bilan d'aménagement du territoire : innovations et blocages dans les Andes centrales (Chili-Pérou-Bolivie) », Mosella, $\mathrm{n}^{\circ}$ spécial « Frontières et Aménagement ». 
Arbaret-Schulz, C., 2002. « Les villes européennes, attracteurs étranges de formes frontalières nouvelles », in B. Reitel, P. Zander, J.-L. Piermay et J.-P. Renard (éds), Villes et frontières, 213-230. Paris : AnthroposEconomica.

Barros, A. v. H., 2004. « Crónica de una etnia anunciada : Nuevas perspectivas de investigación a 10 años de vigencia de la Ley Indígena en San Pedro de Atacama ». Estudios Atacameños (27) : 139-168.

Benedetti, A. et C. Argarañaz, 2003. «Transformations sociales et territoriales à la frontière atacaménienne au cours du $\mathrm{XX}^{\mathrm{e}}$ siècle. Susques : lorsqu'un village dépeuplé devient la 'porte des Andes' ", Revue de Géographie Alpine 91 (3) : 29-45.

BengoA, J., 2000. La emergencia indígena en América Latina. Santiago de Chile : Fondo de Cultura Económica.

Bennafla, K., 1999. "La fin des territoires nationaux ? État et commerce frontalier en Afrique centrale », Politique africaine (73) : 24-48.

Berdichewsky, B., 1986. «Del indigenismo a la indianidad y el surgimiento de una ideología indígena en andinoamérica », América Indigena XLVI (4) : 643-658.

Bouysse-Cassagne, T. et P. Bouysse, 1998. « La montagne vivante dans l'ombre des dieux », in S. Bobbé, Volcans en feu, lieux de vie et de mort, 89-125. Paris : Autrement.

Claval, P., 1985. « Les idéologies spatiales », Cahiers de Géographie du Québec 29 (77) : 261-269.

Darnovsky, M., B. Epstein et R. Flacks (dir.), 1995. Cultural Politics and Social Movements. Philadelphia : Temple University Press.

Demélas, M.-D., 2003. «Estado y actores colectivos. El caso de los Andes », in A. Annino et F.-X. Guerra, Inventando la nación. Iberoamérica siglo XIX, 347-378. México : Fondo de Cultura Económica.

El Correo, Tacna, 23 et 24/3/2002 (Quotidien de Tacna, Pérou).

Elden, S., 2010. « Land, terrain, territory ». Progress in Human Geography (Published online before print April 21, 2010) : 1-19.

Eskelinen, H., I. Liikanen et J. Oksa (eds), 1999. Curtains of Iron and Gold : Reconstructing Borders and Scales of Interaction. Aldershot: Ashgate.

Fontaine, G., 2004. « De l'hacienda au gouvernement. Histoire contemporaine du mouvement indien équatorien », Les cahiers ALHIM (Amérique latine Histoire et Mémoire) 10 (Identités : positionnements des groupes indiens en Amérique latine), http://alhim.revues.org/index110.html

Giraut, F., 2009. «Innovation et territoires : Les effets contradictoires de la marginalité - Innovation and territories : The contradictory effects of marginality », Revue de Géographie Alpine - Journal of Alpine Research 97 (1), http://rga.revues.org/index783.html (version française) ; http://rga.revues.org/index785. html (version anglaise).

GonzÁlez Cortez, H., 1995. Características de la migración campo-ciudad entre los Aymara del Norte de Chile. Arica : Corporación Norte Grande.

González Cortes, H. et H. Gundermann Kroll, 1994. Minorías étnicas e integración subregional. Si Somos Americanos. Iquique : Campus/Universidad Arturo Prat.

Gonzalez Miranda, S., 2000. «Arrieros argentinos en el ciclo salitrero de Tarapacá ». Revista de Estudios Trasandinos 4 : 117-129.

--_-, 2006. Arica y la triple frontera. Integración y conflicto entre Bolivia, Perú y Chile. Iquique : Ed. Aribalo. 2009. « La Presencia Boliviana en la Sociedad del Salitre y la Nueva Definición de la Frontera : Auge y caída de una Dinámica Transfronteriza (Tarapacá 1880-1930) , Chungara Revista de Antropología Chilena (Universidad de Tarapacá, Arica) 41 (1) : 31-40.

González Miranda, S., L. Rouvière et C. Ovando, 2008. « De “Aymaras en la frontera” a "Aymaras sin fronteras". Los gobiernos locales de la triple-frontera andina (Perú, Bolivia y Chile) y la globalización », Diálogo andino $31: 31-46$. 
Grimson, A. (dir.), 1999. Fronteras, nactiones e identidades. La periferia como centro. Buenos Aires-Tucuman : Ciccus/La Crujia.

Gros, C., 2003. « Demandes ethniques et politiques publiques en Amérique latine », Cahiers des Amériques Latines 48 : 11-29. http://www.identidadcolectiva.es/pdf/11.pdf

-_-_, 2007. «Ethniciser la politique, politiser l'ethnicité ? Le dilemme latino-américain », in G. Couffignal, Amérique latine. Les surprises de la démocratie, 91-104. Paris : IHEAL.

Gros, C. et M.-C. Strigler (eds), 2006. Etre indien dans les Amériques. Spoliations et résistances, Mobilisations ethniques et politiques du multiculturalisme. Paris: IHEAL.

Gumuchian, H., E. Grasset et R. Lajarge et al., 2004. Les acteurs, ces oubliés du territoire. Paris : Anthropos/ Economica.

Gundermann Kroll, H., 2000. «Las organizaciones étnicas y el discurso de la identidad en el norte de Chile, 1980-2000 », Estudios Atacameños 19 : 75-91.

2003. « Sociedades indígenas, municipio y etnicidad : La transformación de los espacios políticos locales andinos en Chile », Estudios Atacameños $25: 55-77$.

Halbwachs, M., 1950. La mémoire collective. Paris : PUF.

Hermet, G., 2001. Les populismes dans le monde, une histoire sociologique, XIXe-XXe siècle. Paris : Fayard.

Holland, D., G. Fox et V. Daro, 2008. « Social Movements and Collective Identity : A Decentered, Dialogic View 1 », Anthropological Quarterly 81 (1) Winter 2008 : 95-126.

Joffres, A., 2008. « Le populisme d'Amérique latine en Europe : chronique d'un concept populaire », Nuevo Mundo Mundos Nuevos, Cuestiones del tiempo presente (on line journal). http://nuevomundo.revues. $\operatorname{org} / 3628$

KARASIK, G., 1999. « Tras la genealogía del diablo. Discusiones sobre la nación y el Estado en la frontera argentinoboliviana », in A. Grimson, Fronteras, naciones e identidades ; La periferia como centro, 155-184. Buenos Aires : Ciccus/La Crujia.

Lacroix, L., 2007. « Néoindigénisme d'État et ethnicisation en Bolivie (1994-2005) », in D. Rolland et J. Chassin, Pour comprendre la Bolivie d'Evo Morales, 233-262. Paris : L'Harmattan.

Lamont, M. et C. A. Bail, 2005. « Sur les frontières de la reconnaissance. Les catégories internes et externes de l'identité collective », Revue Européenne des Migrations Internationales (REMI) 21 (2) (Catégorisation et classification, enjeux de pouvoir) : 61-90.

Le Bot, Y., 2006. "Les mouvements indiens en Amérique latine. Étapes, dimensions et significations », in C. Gros et M.-C. Striegler, Etre indien dans les Amériques, 157-168. Paris : Institut des Amériques.

Lévy, J. et M. Lussault (dir.), 2003. Dictionnaire de la géographie et de l'espace des sociétés. Paris : Belin.

LozAno B., C., 2000. « Más allá de la ideología y de la teología : protesta social, vida cotidiana y diferencias culturales en los Andes de Jujuy », Estudios Atacameños 19 : 157-174.

Mariátegui, J. C., 1968. Sept essais d'interprétation de la réalité péruvienne/Siete ensayos de interpretacion de la realidad peruana. Paris : F. Maspero.

Massal, J., 2005. Les mouvements indiens en Équateur. Mobilisations protestataires et démocratie. Paris : Karthala.

Melucci, A., 1996. Challenging Codes. Collective Action in the Information Age. Cambridge : Cambridge University Press.

Morin, F. et B. Saladin d'Anglure, 1995. « L'ethnicité, un outil politique pour les autochtones de l'Arctique et de l'Amazonie », Études/Inuit/Studies 19 (1) : 37-68. 
Murra, J. V., 1992. « Le contrôle vertical d'un nombre maximum d'étages écologiques et le modèle en archipel et quinze ans après, un bilan de la notion d'archipel », in P. Morlon, Comprendre l'agriculture paysanne dans les Andes Centrales. Pérou-Bolivie, 124-140. Paris : INRA.

Murra, J. V. et N. Wachtel, 1978. « Anthropologie Historique des Sociétés Andines », Annales 33 (5-6) : 889-94.

Newman, D. et A. PaAsi, 1998. Fences and neighbours in the postmodern world : boundary narratives in political geography. Progress in Human Geography 22 (2) : 186-207.

Nuñez Atencio, L. et T. S. Dillehay, 1995. Movilidad giratoria, armonía social y desarrollo en los Andes meridionales : patrones de tráfico e interacción económica. Antofagasta, Chile : Universidad Católica del Norte.

PAAsi, A., 1998. « Boundaries as social processes : Territoriality in the world of flows », Geopolitics 3 (1) : 69-88.

--_-, 2009. « Bounded spaces in a 'borderless world': border studies, power and the anatomy of territory ", Journal of Power 2 (2) : 213-234.

Perreault, T., 2003. « Changing Places : Transnational Networks, Ethnic Politics and Community Development in the Ecuadorian Amazon », Political Geography 22 (1) : 61-88.

Radcliffe, S. A., N. Laurie et R. Andolina, 2002. « Reterritorialised Spaces and Ethnic Political Participation : Indigenous Municipalities in Ecuador », Space and Polity 6 (3) : 289-305.

Ricoeur, P., 1986. Idéologie et utopie. Paris : Seuil.

Santana, R. (dir.), 2003. Lo transnacional. Instrumento y desafío para los pueblos indigenas. Quito : Abya-Yala.

TAguiefF, P.-A., 2002. L'illusion populiste, de l'archaïsme au médiatique. Paris : Berg International.

Van Kessel, J., 1980. Holocausto al progreso. Los Aymarás de Tarapaca.' Amsterdam : Centrum voor Studie en Documentatie van Latijns-Amerika.

WAChtel, N., 1992. « Note sur le problème des identités collectives dans les Andes méridionales », L'Homme 32 $(122-124): 39-52$. 